\title{
VITAMIN D ASSOCIATION; MAJOR RISK FACTORS OF CARDIOVASCULAR DISEASE
}

1. MBBS, FCPS

Assistant Professor Department of Medicine,

LUMHS, Jamshoro, Sindh, Pakistan

2. MBBS, (FCPS trainee cardiology)

LUMHS, Jamshoro, Sindh, Pakistan

Correspondence Address:

Dr. Ambreen Haidar

Postal address:

$\mathrm{C} /$ of - national medical

Center near national CNG khurshid

Town hala naka Hyderabad

dr.sajidarain@gmail.com

Article received on: 25/02/2015

Accepted for publication: 06/07/2015

Received after proof reading: $12 / 10 / 2015$

\section{Dr. Sayed Fasih Ahmed Hashmi ${ }^{1}$, Dr. Ambreen Haidar ${ }^{2}$}

ABSTRACT... OBJECTIVE: The target of this study to focus, vitamin D level is the significant risk factors for the patients with cardio vascular disease at Liaquat University Hospial Hyderabad / Jamshoro. Materialand Methods: This observational study was done at cardiology department of Liaquat University Hospital Hyderabad. All the cases with history of congenital heart disease, pregnancy, malignancy, renal failure and chronic liver disease, were excluded from the study. Complete medical history was taken, and major risk factors of the cardiovascular disease including, diabetes, smoking, family history, hypertension, hypercholestremia, dyslipidemia and history of alcohol consumption and others were documented. Serum VD level was tested by blood samples from research laboratory of Liaquat Medical hospital Hyderabad, and all the results were noted on the Performa according to the risk factors. Consequences of VD level were arranged by criteria, that's taken from the study of Satish Karur etal. ${ }^{10}$ Results: Total 100 patients were incorporated in the study with the mean age of $48.2+12.4$. Male were found in the majority. According to distribution of heart disease of his study ischemic heart disease had noted commonest58\%. In the hypertensive patients VD deficiency had noted in $39.63 \%$. In smoker patients VD deficiency was noted in $52.77 \%$. In patients with hypercholestremia $50.0 \%$, Alcoholic patients were found with deficiency of VD were $50.0 \%$. In patients with obesity $57.14 \%$. In the diabetic patients deficiency $15.0 \%$, insufficiency $50.0 \%$ and sufficiency was seen $35.0 \%$. In the Patients of dyslipidemia deficiency $5.0 \%$, insufficiency $50.0 \%$ and sufficiency was seen $45.0 \%$. Patients with family history of cardiovascular disease having deficiency $25.0 \%$ of the cases, insufficiency $12.50 \%$ while sufficiency in $62.50 \%$ in the cases. Conclusion: In this study we concluded that VD deficiency in one of the major risk factor for cardiovascular disease; its possible association was found in this study with many risk factors of heart diseases. Like our study there is very need of experimental and prospective more studies, to find out the mechanism undergoing increasing cardiovascular risk, and prevent the cardiovascular disease.

Key words: $\quad$ Cardiovascular disease (CVD),

Article Citation: Hashmi SFA, Haidar A. Vitamin D association; major risk factors of cardiovascular disease. Professional Med J 2015;22(10):1316-1320. DOI:10. $17957 / T P M J / 15.2959$

\section{INTRODUCTION}

Cardiovascular sicknesses additionally called ischemic heart disease, stroke, and peripheral vascular disease. CVD hardly associated with way of life, particularly the utilization of tobacco, poor habits of diet, physical activity, and psychosocial stress. ${ }^{1}$ Risk factors of the CVD such as; hypertension, dyslipidemia, obesity, diabetes mellitus, and in addition myocardial infarction, coronary artery disease or stroke, are the most predominant illnesses and record for the real reasons for death around the world, particularly in Western countries. ${ }^{2}$ The revelation that the VD receptor (VDR) is universally communicated in all body cells, for example, resistant, vascular or myocardial cells, proposes an association of VD -intervened impacts in a few different frameworks separated from musculoskeletal tissues. ${ }^{2}$ This has prompted far reaching research on VD as a potential impacting consider the pathogenesis of a few unending non-skeletal illness, such as infectious or auto immune disease, growth or cardiovascular infections (CVD). ${ }^{4,5}$

VD insufficiency is connected with expanded risks for cardiovascular disease (CVD) and death. ${ }^{6} A$ relationship of VD and several CV risk factors and illness has been widely assessed among the most 
recent years. Various observational studies, observational meta-analysis, and additionally some interventional studies have tended to the possible linkage of VD inadequacy and the increased CVD and its risk factors. ${ }^{7,8}$ Accordingly purpose of this study to focus VD level in the significant risk factors in the cases with cardiovascular diseases at cardiology department of Liaquat University Hospital Hyderabad / Jamshoro.

\section{Data Collection Procedure}

This observational study was carried out at cardiology department of LUH Hyderabad. Study was done with the duration of time from June 2014 to November 2014. Total 100 cases were selected in this study including both genders. All the cases were included abovethe age of 20 years. All the patients were selected; those were admitted in the cardiology department with cardiovascular disease mostly hypertension, ischemic heart disease, stroke, heart failure and rheumatic heart disease including coronary artery disease. All the cases with the history of congenital heart disease, pregnancy, malignancy, renal failure and chronic liver disease, were excluded from the study. Complete history was taken, and major risk factors of the cardiovascular disease including, diabetic, smoking, family history, hypertension, hypercholestremia, dyslipidemia and history of alcohol consumption and others were recorded. Serum VD level was tested by blood samples from research and diagnostic laboratory of LUH Hyderabad, and all the results were noted on the Performa according to the risk factors. Results of VD level were classified according to criteria which was taken from the study of Satish Karur etal. ${ }^{10}$

\begin{tabular}{|c|c|}
\hline Serum $25(\mathbf{O H}) \mathbf{D}(\mathbf{n g} / \mathbf{m l})$ & VD status. \\
$<20$ & Deficient \\
$21-29$ & Insufficient \\
$\geq 30$ & Sufficient \\
\hline
\end{tabular}

Table-I. Status of VD.

\section{RESULTS}

Total 100 patients had integrated in the study with the mean age of $48.2 \pm 12.4$. Male were found in the majority with $73 \%$ while females were noted $27 \%$. The most common age group of this study was noted 41 to 50 years of the age $51 \%$ and the second most common age group was found between 30 to 40 years of the age. Majority of the patients recorded areas. Table-II.

\begin{tabular}{|c|c|c|}
\hline Characteristics & Frequency & \%age \\
\hline Age groups & & \\
$20-30$ & 02 & $2.0 \%$ \\
$31-40$ & 31 & $31.0 \%$ \\
$41-50$ & 51 & $51.0 \%$ \\
$51-60$ & 11 & $11.0 \%$ \\
$>60$ & 05 & $5.0 \%$ \\
Gender & & \\
Male & 73 & $73.0 \%$ \\
Female & 27 & $27.0 \%$ \\
Residence & & \\
Rural & & \\
Urban & 41 & $41.0 \%$ \\
\hline
\end{tabular}

Table-II. Demographic characteristics. $(n=100)$

According to distribution of heart disease of his study ischeming heart disease was seen most common $58 \%$, while stroke, rheumatic heart disease and heart failure were found with the percentage of $11 \%, 9 \%$ and $6 \%$ respectively. Figure-l.

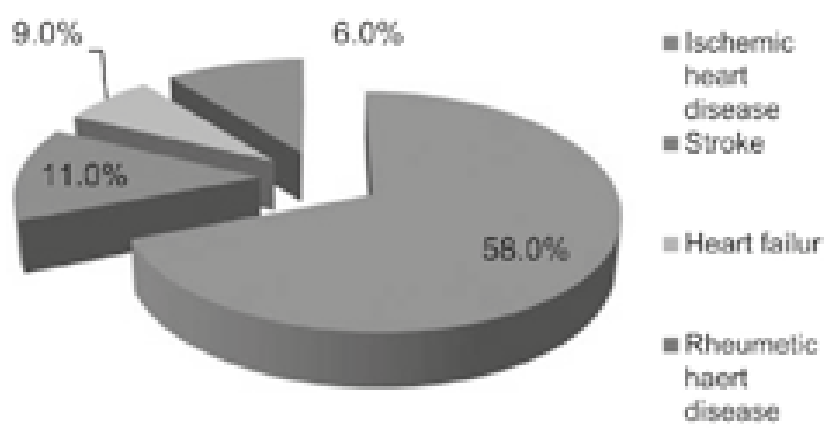

Figure-I. Prevalence of Cardiovascular disease. $\mathrm{N}=100$

In the hypertensive patients VD deficiency was noted in $39.63 \%$, insufficiency $35.85 \%$ and sufficiency was seen $24.52 \%$.In smoker patients VD deficiency was noted in $52.77 \%$, insufficiency $33.33 \%$ and sufficiency was seen $13.0 \%$. In the cases with hypercholestremia VD deficiency was recorded in $50.0 \%$, insufficiency $25.0 \%$ and sufficiency was noted $25.0 \%$. Alcoholic patients were seen with deficiency of VD was $50.0 \%$, insufficiency was $25.0 \%$ and sufficiency was seen $25.0 \%$. Patients with obesity were noted with VD deficien- 
cy was noted in $57.14 \%$, insufficiency $14.28 \%$ and sufficiency was seen $28.58 \%$.In the diabetic patients VD deficiency was noted $15.0 \%$, insufficiency $50.0 \%$ and sufficiency was seen $35.0 \%$.In the Patients of dyslipidemia VD deficiency was found in $5.0 \%$, insufficiency $50.0 \%$ and sufficiency was seen $45.0 \%$. Patients with family history of cardiovascular disease having VD deficiency $25.0 \%$ of the cases, insufficiency $12.50 \%$ while sufficiency in $62.50 \%$ in the cases. Table-III.

\begin{tabular}{|c|c|c|c|c|}
\hline Risk factors & Defi. $<20$ & Insuffi. 21-30 & Suffi. $>\mathbf{3 0}$ & Total/ \% \\
\hline & No. of $\mathrm{pt} / \%$ & No. of pt/\% & No. of pt/\% & No. of $\mathrm{pt} / \%$ \\
\hline $\begin{array}{l}\text { Hypertension } \\
\text { Smoking Hypercholestremia } \\
\text { Alchohal } \\
\text { Obesity } \\
\text { Diabetes } \\
\text { Dyslipidemia } \\
\text { Family history }\end{array}$ & $\begin{array}{c}21 / 39.63 \% \\
19 / 52.77 \% \\
20 / 50.0 \% \\
10 / 50.0 \% \\
08 / 57.14 \% \\
03 / 15.0 \% \\
01 / 5.0 \% \\
04 / 25.0 \%\end{array}$ & $\begin{array}{c}19 / 35.85 \% \\
12 / 33.33 \% \\
10 / 25.0 \% \\
5 / 25.0 \% \\
02 / 14.28 \% \\
10 / 50.0 \% \\
10 / 50.0 \% \\
02 / 12.50 \%\end{array}$ & $\begin{array}{c}13 / 24.52 \% \\
05 / 13.0 \% \\
10 / 25.0 \% \\
05 / 25.0 \% \\
04 / 28.58 \% \\
07 / 35.0 \% \\
09 / 45.0 \% \\
10 / 62.50 \%\end{array}$ & $\begin{array}{l}53 / 100 \% \\
36 / 100 \% \\
40 / 100 \% \\
20 / 100 \% \\
14 / 100 \% \\
20 / 100 \% \\
20 / 100 \% \\
16 / 100 \%\end{array}$ \\
\hline
\end{tabular}

\section{DISCUSSION}

This observational data recommended that $\mathrm{D}$ deficiency is highly linked to developed CVD risk. In the study of Naeem Imran abbasi et al, ${ }^{11}$ reported that male in the majority as compare to females with the percentage of male $65 \%$ and female $35 \%$, in his study most common age group was seen 41-50 years and second most common age group above 60 years. Another study from Karachi in tertiary care hospital reported majority of the males with cardiovascular diseases. ${ }^{12}$ Similarly in this study male were found in the majority with $73 \%$ while females were noted $27 \%$. The important age group of this study was noted 40 to 50 years of the age $51 \%$ and the $2^{\text {nd }}$ commonest age group was noted between 30 to 40 years of the age.

Like as are view prevalence of in Hong Kong, Taiwan, South Korea, Singapore, Malaysia, Thailand, Philippines and Indonesia, reported showed that frequency of ischemic and the hemorrhagic strokes different from $17 \%$ to $33 \% .{ }^{13}$ Some other series as of countries of Asia demonstrated incidence of $\mathrm{ICH}$ is above than in Caucasians with just about $20 \%$ to $30 \%$ being hemorrhagic. ${ }^{13,14} \mathrm{~A}$ study conducted at Umm Al Qura University reported prevalence of cardiovascular disease as, stroke $17.54 \%$, ischemic heart disease $33.33 \%$, heart failure $15.79 \%$ and cardiac myopathy $8.77 \% .{ }^{15}$ While in the present study ischemic heart disease was seen most common $32 \%$, second most common was acute MI $26 \%$ while stroke, rheumatic heart disease and heart failure were found with the percentage of $11 \%, 9 \%$ and $6 \%$ respectively.

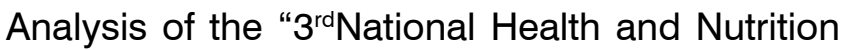
Examination Survey (NHANES III) demonstrated lower $25(\mathrm{OH})-\mathrm{D}$ ratio were linked to higher $\mathrm{BP}$ in a representative sample of population of U.S. through cultural difference in $25(\mathrm{OH})$-D ratio b/w black and white cases. ${ }^{16}$ In the study of forman JP et al. ${ }^{17}$ demonstrated that potential linkage among measured plasma $25(\mathrm{OH})-\mathrm{D}$ ratio levels and consequential cause of increasing hypertension. Similarly in the present series VD deficiency was noted in $39.63 \%$ and insufficiency $35.85 \%$ of the patients. In the study of Satis karur et al, ${ }^{11}$ reported that VD deficiency in the smoking patients $53 \%$, in the alcoholic patients $1 \%$, in diabetic patients $50 \%$, in the patients high level of cholesterol $72 \%$ and in the hypertensive patients $35 \%$. As well as the present study also showing similar results. In smoker patients VD deficiency was noted in $52.77 \%$, insufficiency $33.33 \%$ and sufficiency was seen $13.0 \%$. In the cases with hypercholestremia VD deficiency was recorded in $50.0 \%$, insufficiency $25.0 \%$ and sufficiency was noted $25.0 \%$.

In the study of Ayman El-Menyar et al, ${ }^{18}$ reported that VD deficiency in cardiac smoker male patients $7 \%$ and in the female patients $9 \%$, in male diabetic patients deficiency in $38 \%$ while in females having $22 \%$,further more in this study 
showed VD deficiency in the male patients with dyslipidemia $41 \%$ while in the females $29 \%$. Similarly in this study alcoholic patients were seen with deficiency of VD was $50.0 \%$, insufficiency was $25.0 \%$ and sufficiency was seen $25.0 \%$. Patients with obesity were noted with VD deficiency was noted in $57.14 \%$, insufficiency $14.28 \%$ and sufficiency was seen $28.58 \%$. In the diabetic patients VD deficiency was noted $15.0 \%$, insufficiency $50.0 \%$ and sufficiency was seen $35.0 \%$. In the Patients of dyslipidemia VD deficiency was found in $5.0 \%$, insufficiency $50.0 \%$ and sufficiency was seen $45.0 \%$. Patients with family history of cardiovascular disease having VD deficiency $25.0 \%$ of the cases, insufficiency $12.50 \%$ while sufficiency in $62.50 \%$ in the cases. Thomas $\mathrm{J},{ }^{19}$ also showed comparable result of VD deficiency in different risk factors of cardiovascular disease.

\section{CONCLUSION}

In this study we concluded that VD deficiency in one of the risk factor for cardiovascular disease; it is also increase by other cardiac risk factors, because it is associated with many risk factors of cardiovascular disease in our study. Like our study there is very need of experimental and prospective more studies, to find out the mechanism undergoing increasing cardiovascular risk, and prevent the cardiovascular disease.

Copyright@ 06 July, 2015.

\section{REFRENCES:}

1. World Health Organization. Joint WHO/FAO Expert Consultation on Diet, Nutrition and the Prevention of Chronic Diseases. 2002. Report No. 916.

2. Organization, W.H. World Health Statistics 2012; World Health Organization: Geneva, Switzerland, 2012.

3. Bouillon, R.; Carmeliet, G.; Verlinden, L.; van Etten, E.; Verstuyf, A.; Luderer, H.F.; Lieben, L.; Mathieu, C.; Demay, M. VD and human health: Lessons from VD receptor null mice.Endocr. Rev. 2008, 29, 726-776.

4. Souberbielle, J.-C.; Body, J.-J.; Lappe, J.M.; Plebani, M.; Shoenfeld, Y.; Wang, T.J.; Bischoff-Ferrari, H.A.; Cavalier, E.; Ebeling, P.R.; Fardellone, P.; et al. VD and musculoskeletal health, cardiovascular disease, autoimmunity and cancer: Recommendations for clinical practice.Autoimmun. Rev. 2010,9, 709-715.
5. Pilz, S.; Tomaschitz, A.; März, W.; Drechsler, C.; Ritz, E.; Zittermann, A.; Cavalier, E.; Pieber, T.R.; Lappe, J.M.; Grant, W.B.; et al. VD , cardiovascular disease and mortality.Clin. Endocrinol. (Oxf.) 2011, 75, 575-584

6. Michos ED, Melamed ML: VD and cardiovascular disease risk. Curr Opin Clin Nutr Metab Care.2008;11: 7-12.

7. Burgaz, A.; Orsini, N.; Larsson, S.C.; Wolk, A. Blood 25-hydroxyVD concentration and hypertension: $A$ meta-analysis.J. Hypertens. 2011, 29, 636-645.

8. Zittermann, A.; lodice, S.; Pilz, S.; Grant, W.B.; Bagnardi, V.; Gandini, S. VD deficiency and mortality risk in the general population: A meta-analysis of prospective cohort studies.Am. J. Clin. Nutr. 2012, 95, 91-100.

9. Grandi, N.C.; Breitling, L.P.; Brenner, H. VD and cardiovascular disease: Systematic review and meta-analysis of prospective studies.Prev. Med. 2010, 51, 228233.

10. Satish Karur, Virupakshappa Veerappa, Manjunath C. Nanjappa. Study of VD deficiency prevalence in acute myocardial infarction. IJC Heart \& Vessels.2014;3; 5759

11. Imran Naeem Abbasi, Deedar Hussain Gajoo, Sunil Kumar, Saima Zainab, Zafar Fatmi. Pattern of cardiovascular diseases according to age and gender in a rural district of Pakistan.Pak Heart J 2013 Vol. 46 (03) : 178-183

12. Abbas $\mathrm{S}$. Disease burden of ischemic heart diseases in Pakistan and its risk factors. Ann Pak Inst Med Sci. 2009;5:145-50.

13. Asian Acute Stroke Advisory Panel. Stroke epidemiological data of nine Asian countries. J Med Assoc Thai. 2000; 83, 1-7

14. Kay R, Woo J, Kreel L, Wong HY, Teoh R, NicholIs MG. Stroke subtypes among Chinese living in Hong Kong: the Shatin Stroke Registry. Neurology JID.1992;42:985-87.

15. Waleed H. Almalki. The prevalence of cardiovascular diseases and role of protective measures among hajj pilgrims 1432 (2011). Pakistan Journal of Pharmacology. 2012;29;2:29-34

16. Scragg R, Sowers M, Bell C. Serum 25-hydroxy VD, ethnicity, and blood pressure in the Third National Health and Nutrition Examination Survey. Am J Hypertens. 2007;20(7):713-719

17. Forman JP, Giovannucci E, Holmes MD, et al. Plasma 25- hydroxyVD levels and risk of incident hyperten- 
sion. Hypertension. 2007;49(5):1063-1069

18. Ayman El-Menyar*,1,2, Ali Rahil3, Khalid Dousa3, Walid Ibrahim3, Talal Ibrahim3, Rasha Khalifa3,and Mohamed Osman Abdel Rahman. Low VD and Cardiovascular Risk Factors in Males and Femalesfrom a Sunny, Rich Country. The Open Cardiovascular Medicine Journal, 2012, 6, 76-80
19. Thomas J. Wang, MD; Michael J. Pencina, PhD; Sarah L. Booth, PhD; Paul F. Jacques, DSc;Erik Ingelsson, $\mathrm{MD}, \mathrm{PhD}$; Katherine Lanier, BS; Emelia J. Benjamin, MD, MSc;Ralph B. D'Agostino, PhD; Myles Wolf, MD, MMSc*; Ramachandran S. Vasan, MD*.VD Deficiency and Risk of Cardiovascular Disease. Circulation. 2008;117:503-511.

\section{PREVIOUS RELATED STUDY}

Ahmed Bilal, Muhammad Owais Fazal, Fraz Saeed Qureshi, Muqqadas Shaheen, Muhammad Irfan Iqbal, Sadia Khan, Abdullah Bin Saeed. SERUM VITAMIN D DEFICIENCY, A NEW EPIDEMIC (Original) Prof Med Jour 17(1) 111-116 Jan, Feb, Mar 2010.

Muzaffar Mehmood Khan, Muhammad Tahir, Mohsin Raza, Muhammad Ali Bhatti, Muhammad Riaz Khokhar. Hepatitis C; association of interferon-ribavirin therapy with hearing loss (Original) Professional Med J Mar-Apr 2012;19(2): 193-196.

Rizwana Kitchlew, Fuad Ahmad Siddiqi, Asif Hshmi. Hepatitis C (Original) Professional Med J May-Jun 2012;19(3): 375-381.

\section{"Champions are willing to do what they hate, In order to get what they love."}

\section{Unknown}

\section{AUTHORSHIP AND CONTRIBUTION DECLARATION}

\begin{tabular}{|c|l|l|l|}
\hline Sr. \# & \multicolumn{1}{|c|}{ Author-s Full Name } & \multicolumn{1}{|c|}{ Contribution to the paper } & Author=s Signature \\
1 & $\begin{array}{l}\text { Dr. Sayed Fasih Ahmed } \\
\text { Hashmi }\end{array}$ & 1st Author \\
2 & $\begin{array}{l}\text { Dr. Ambreen Haider } \\
\text { 2nd Author }\end{array}$ \\
\hline
\end{tabular}

